\title{
A NORMAL FIRST COUNTABLE ccc NONSEPARABLE SPACE
}

\author{
MURRAY G. BELL
}

\begin{abstract}
We construct an absolute example of a space having the properties in the title. Let $Y$ be the set of nonempty finite subsets of the Cantor cube of countable weight. The Pixley-Roy topology on $Y$ is not normal, but the Vietoris topology on $Y$ is normal. Our space can be considered a normalization of the Pixley-Roy topology on $Y$ by adding cluster points which as a subspace have the Vietoris topology. The Alexandroff duplicating procedure is used liberally to glue the space together. The example is also a sigma compact paracompact $p$-space.

If further set-theoretic assumptions are made (e.g. $V=L$ or $\mathrm{MA}+$ $\neg \mathrm{CH}$ ), then it is known that even perfectly normal such examples exist.
\end{abstract}

0. Introduction. A space $X$ is ccc if each collection of disjoint open sets is at most countable. A Souslin line is a ccc nonseparable linearly ordered topological space. It is consistent with the usual axioms for set theory (i.e. ZFC) that a Souslin line exists (Jech [7]). Such a line yields an example of a perfectly normal first countable ccc nonseparable space (cf. Rudin [11]). Martin's axiom plus the negation of the continuum hypothesis is also consistent with the usual axioms (Solovay and Tennenbaum [12]). It implies that there are no Souslin lines, nonetheless, one is still able to construct a perfectly normal first countable ccc nonseparable space (Przymusiński and Tall [10]). One is naturally led to wondering whether an absolute example with such properties can be constructed in ZFC alone. According to a theorem of F. Tall in [13], the existence of such a space implies either the existence of a hereditarily Lindelöf nonseparable regular $T_{2}$ space or the existence of a nonmetrizable normal Moore space. Neither of these problems has been satisfactorily answered yet.

A less grandiose endeavour would be to construct an absolute example of a normal first countable ccc nonseparable space. As far as the author knows, no such absolute example exists in the literature to date. The example $X^{*}$ that we construct is also sigma compact and is a perfectpreimage of a metrizable space.

1. Two special topologies. Let $X$ be a $T_{1}$ space with topology $\tau$ and let $C\langle X\rangle$ be the space of all nonemtpy closed subsets of $X$ endowed with the Vietoris topology [15]. If $\theta$ is a finite subcollection of $\tau$, define $\langle\theta\rangle=\{Y \in C\langle X\rangle$ :

Received by the editors May 1, 1978 and, in revised form, on June 16, 1978. AMS (MOS) subject classifications (1970). Primary 54D15; Secondary 54G20.

Key words and phrases. First countable, normal, ccc, separable. 
$Y \subseteq \cup \theta$ and for each $O \in \theta, Y \cap O \neq \varnothing\}$. The collection $\{\langle\theta\rangle: \theta$ is a finite subcollection of $\tau$ \} serves as a base for the topology on $C\langle X\rangle$. The subspace of $C\langle X\rangle$ consisting of all finite subsets of $X$ will be denoted by $F\langle X\rangle$.

Another useful topology on the nonempty closed subsets of $X$, denoted by $C[X]$, is the Pixley-Roy topology [9]. If $K \in C[X]$ and $O \in \tau$, then define $[K, O]=\{G \in C[X]: K \subseteq G \subseteq O\}$. The collection $\{[K, O]: K \in C[X]$ and $O \in \tau\}$ serves as a base for the topology on $C[X]$. The subspace of $C[X]$ consisting of all finite subsets of $X$ will be denoted by $F[X] . F[X]$ has a finer topology then $F\langle X\rangle$. An excellent reference for facts about both $F[X]$ and $F\langle X\rangle$ (and more) is E. van Douwen [3].

2. Construction of $X^{*}$ and its properties. From now on, $X$ will denote the Cantor cube of weight $\omega$, i.e. $X=[D(2)]^{\omega}$, where $\omega$ is the first infinite ordinal and $D(2)$ is the discrete space of cardinality 2 . Let $\mathscr{F}(X)$ be the collection of all nonempty finite subsets of $X$ and let $\mathscr{F}^{*}(X)=\mathscr{F}(X) \cup\{\varnothing\}$. The collection of all clopen subsets of $X$ is denoted by $\mathscr{B}$ and is a base for the topology on $X$. We denote the collection of all finite subsets of $\mathscr{B}$ by $\mathscr{B} *$.

If $\theta \in \mathscr{B}^{*}$, then define $\langle\theta\rangle=\left\{F \in \mathcal{F}^{*}(X): F \subseteq \cup \mathcal{\theta}\right.$ and for each $O \in \theta, F \cap O \neq \varnothing\}$. Note that $\varnothing \in\langle\theta\rangle$ iff $\theta=\varnothing$. If $F \in \mathscr{F}^{*}(X)$ and $O \in \mathscr{B}$, then define $[F, O]=\left\{G \in \mathscr{F}^{*}(X): F \subseteq G \subseteq O\right\}$.

We now define the space $X^{*}$.

$$
X^{*}=\left\{(F, G): F \in \mathscr{F}^{*}(X), G \in \mathcal{F}^{*}(X) \text { and } F \cap G=\varnothing\right\} .
$$

Henceforth, whenever we write $(F, G)$ it is understood that $F \cap G=\varnothing$. A basic neighbourhood of $(F, G)$ has the form $V[(F, G), \theta]=\{(H, K): H \cup K$ $\in\langle\theta\rangle$ and $K \in[G, \cup \theta-F]\}$ where $\theta \in \mathscr{B}^{*}$ and $F \cup G \in\langle\theta\rangle$. It is straightforward to show that $V\left[(F, G), \Theta_{1} \mathcal{O}_{2}\right] \subseteq V\left[(F, G), \Theta_{1}\right] \cap V[(F, G)$, $\left.\mathcal{O}_{2}\right]$ where $\mathcal{O}_{1} \cap \mathcal{O}_{2}=\left\{O_{1} \cap O_{2}: O_{1} \in \mathcal{O}_{1}, O_{2} \in \mathcal{O}_{2}\right.$ and $\left.O_{1} \cap O_{2} \neq \varnothing\right\}$.

To verify that this is a valid neighbourhood assignment to the points of $X^{*}$, it suffices now to show that each basic neighbourhood contains a basic neighbourhood of each of the points that it contains. Let $V[(F, G), \theta]$ be a basic neighbourhood of $(F, G)$. If $(H, K) \in V[(F, G), \theta]$, then $H \cup K \in$ $\langle\theta\rangle$ and $K \in[G, \cup \theta-F]$. Choose $\theta^{\prime} \in \mathscr{B}^{*}$ such that $H \cup K \in\left\langle\theta^{\prime}\right\rangle \subseteq$ $\langle\theta\rangle$ and $\cup \theta^{\prime}-H \subseteq \cup \mathcal{O}^{\prime}-F$. Then, $(H, K) \in V\left[(H, K), \theta^{\prime}\right] \subseteq$ $V[(F, G), \mathcal{O}]$.

The point $(\varnothing, \varnothing)$ is the only isolated point in $X^{*}$. To motivate our choice of such a topology, let us look more closely at three special subspaces of $X^{*}$. Define

$$
\begin{aligned}
& P=\{(\varnothing, G): G \in \mathscr{F}(X)\}, \\
& A=\{(F, G):|F|+|G|=1\}, \\
& V=\{(F, \varnothing): F \in \mathscr{F}(X)\} .
\end{aligned}
$$

As subspaces of $X^{*}, P$ is homeomorphic to $F[X], A$ is homeomorphic to the Alexandroff double of $X$ (cf. Willard [16]), and $V$ is homeomorphic to $F\langle X\rangle$. 
Furthermore, if we define, for each $G \in \mathcal{F}^{*}(X)$ and each $n \geqslant|G|, A(G, n)=$ $\{(\varnothing, H): G \subseteq H$ and $|H|=n\} \cup\{(H, G):|H|=n-|G|\}$, then $A(G, n)$ is homeomorphic to the Alexandroff double of the subspace $\{H \in F\langle X-G\rangle$ : $|H|=n-|G|\}$.

A. $X^{*}$ is 0 -dimensional. We show that each $V[(F, G), \theta]$ is closed in $X^{*}$. Assume that $(H, K) \notin V[(F, G)$, $\theta]$. If $H \cup K \notin\langle\theta\rangle$, then choose $\theta^{\prime} \in$ $\mathscr{B}^{*}$ such that $H \cup K \in\left\langle\theta^{\prime}\right\rangle$ and $\left\langle\theta^{\prime}\right\rangle \cap\langle\theta\rangle=\varnothing$. If $H \cup K \in\langle\theta\rangle$, but $K \notin[G, \cup \theta-F]$, then choose $\theta^{\prime} \in \mathscr{B}^{*}$ such that $H \cup K \in\left\langle\theta^{\prime}\right\rangle$ and $\cup \vartheta^{\prime} \cap[G-(H \cup K)]=\varnothing$. In either case, $V\left[(H, K), \theta^{\prime}\right] \cap V[(F, G), \theta]$ $=\varnothing$.

B. $X^{*}$ is first countable. This follows from the fact that the finite subsets of $X$ have countable neighbourhood bases.

C. $X^{*}$ is $T_{1}$ (and therefore $T_{2}$ ). Each point in $X^{*}$ is the intersection of its clopen basic neighbourhoods.

D. The mapping $f: X^{*} \rightarrow F\langle X\rangle$ defined by $f((F, G))=F \cup G$ is perfect.

(i) Let us check that $f$ is continuous. If $\langle\vartheta\rangle$ is a basic open set of $F\langle X\rangle$, then

$$
\begin{aligned}
f^{-1}\langle\theta\rangle & =\{(F, G): F \cup G \in\langle\theta\rangle\} \\
& =\cup\{V[(F, G), \theta]: F \cup G \in\langle\theta\rangle\}
\end{aligned}
$$

which is open in $X^{*}$.

(ii) Let us check that $f$ is closed. Let $C$ be closed in $X^{*}$. Take $F \in F\langle X\rangle-$ $f(C)$. Then, $\{(A, F-A): A \subseteq F\} \cap C=\varnothing$. For each $A \subseteq F$, choose an $\vartheta_{A} \in \mathscr{B}^{*}$ such that $F \in\left\langle\vartheta_{A}\right\rangle$ and $V\left[(A, F-A), \theta_{A}\right] \cap C=\varnothing$. Choose $\theta \in \mathscr{B}^{*}$ such that $F \in\langle\theta\rangle \subseteq \cap_{A \subseteq F}\left\langle\theta_{A}\right\rangle$. Then $\langle\theta\rangle \cap f(C)=\varnothing$; because, if $R \in\langle\theta\rangle \cap f(C)$, then $R=A \cup B$ where $(A, B) \in C$, and this means that $(A, B) \in V\left[(F-B, F \cap B), \mathcal{O}_{F-B}\right] \cap C$, which is a contradiction.

(iii) $f$ is clearly a finite-to-one map.

Since $X$ is compact, it follows from a theorem due to $\mathrm{L}$. Vietoris [15] that $C\langle X\rangle$ is compact. For each $n<\omega,\{F \in C\langle X\rangle:|F| \leqslant n\}$ is a closed subspace of $C\langle X\rangle$. Therefore, $F\langle X\rangle$ is sigma compact (a union of countably many compact subspaces). Since sigma compactness is preserved under perfect preimages, we have that $X^{*}$ is sigma compact. Since $X$ is compact and metrizable, $C\langle X\rangle$ is metrizable (cf. Michael [8]) and therefore $F\langle X\rangle$ is metrizable. The space $X^{*}$, being a perfect preimage of $F\langle X\rangle$, is a paracompact $p$-space. Such spaces are investigated by A. Arhangel'skii in [1].

E. $X^{*}$ is ccc. As noted before, $P$ is homeomorphic to $F[X]$. E. van Douwen [3] has shown that for every $T_{1}$ space $Y, d(\beta F[Y])=\operatorname{nw}(Y)$, where $d$ is the density character, $\beta F[Y]$ is the Stone-Čech compactification of $F[Y]$ and $\mathrm{nw}(Y)$ is the smallest cardinal of a network for $Y$ (a collection of nonempty subsets $\Re$ of a space $Y$ is called a network if for each $y \in Y$ and each neighbourhood $U$ of $y, y \in N \subseteq U$ for some $N \in \mathfrak{\Re}$ ). Since $X$ has a 
countable network, it follows that $\beta F[X]$ is separable and thus that $F[X]$ is ccc. Since $P \cup\{(\varnothing, \varnothing)\}$ is dense in $X^{*}, X^{*}$ is ccc.

F. $X^{*}$ is not separable. In fact, $d\left(X^{*}\right)=c$ where $c=\left|[D(2)]^{\omega}\right|$. Assume $Y$ is a dense subset of $X^{*}$ and $|Y|<c$. Choose $K \in \mathscr{F}(X)$ such that for all $(A, B) \in Y, K \nsubseteq B$. Then $(\varnothing, K) \notin \mathrm{Cl}_{X^{*}} Y$, since $V[(\varnothing, K),\{X\}] \cap Y=\varnothing$. Hence, any dense subset of $X^{*}$ has cardinality $c$.

Thus, we see that $X^{*}$ is a first countable, 0 -dimensional, sigma compact (therefore normal) ccc nonseparable space. This is most surprising when we realize that $X^{*}$ admits a finte-to-one perfect map onto a metric space.

3. Concluding remarks. Since $\beta F[X]$ is separable, it follows (see van Douwen [4]) that every compactification of $F[X]$ is separable. Hence every compactification of $X^{*}$ is separable. In particular, $d\left(\beta X^{*}\right) \neq d\left(X^{*}\right)$. Since $X^{*}$ is a paracompact $p$-space, this answers negatively a question raised by van Douwen in [4]. The author thanks Jan van Mill for bringing this to his attention. Furthermore, since $X^{*}$ is not separable, it follows that $X^{*}$ is a first countable Lindelöf space with no first countable compactification. Examples of such a phenomenon were previously given by Ul'janov [14] and van Douwen and Przymusiński [5].

A. Hajnal and I. Juhász [6] have shown that it is consistent with ZFC that every first countable compact ccc space be separable. This shows us that our example cannot be made locally compact in ZFC alone.

The author was initially motivated by an open question stated in [2]. Namely, is there a "real" example of a first countable normal ccc space with no dense Lindelöf subspace? Unfortunately, $X^{*}$ is Lindelöf. The idea was to find a first countable normalization of $F[X]$ which was not Lindelöf. This question is still open.

\section{REFERENCES}

1. A. V. Arhangel'skii, On a class of spaces containing all metric spaces and all locally bicompact spaces, Amer. Math. Soc. Transl. (2) 92 (1970), 1-39.

2. M. Bell, J. Ginsburg and G. Woods, Cardinal inequalities for topological spaces involving the weak Lindelö number, Pacific J. Math. (to appear).

3. E. van Douwen, The Pixley-Roy topology on spaces of subsets, Set-Theoretic Topology, ed. G. M. Reed, Academic Press, New York, 1977.

4. , Density of compactifications, Set-Theoretic Topology, ed. G. M. Reed, Academic Press, New York, 1977.

5. E. van Douwen and T. C. Przymusinski, First countable and countable spaces all compactifications of which contain $\beta N$, Fund. Math. (to appear).

6. A. Hajnal and I. Juhász, A consequence of Martin's Axiom, Indag. Math. 33 (1971), 457-463.

7. T. Jech, Nonprovability of Souslin's Hypothesis, Comment. Math. Univ. Carolinae 8 (1967), 293-296.

8. E. Michael, Topologies on spaces of subsets, Trans. Amer. Math. Soc. 71 (1951), 152-183.

9. C. Pixley and P. Roy, Uncompletable Moore spaces, in Proc. Auburn Topology Conference, ed. W.R.R. Transue, (Auburn, Alabama, 1969).

10. T. Przymusinski and F. D. Tall, The undecidability of the existence of a non-separable normal Moore space satisfying the countable chain condition, Fund. Math. 85 (1974), 291-297. 
11. M. E. Rudin, Lectures on set-theoretic topology, CBMS Regional Conference Ser. in Math. no. 23, Amer. Math. Soc., Providence, R.I., 1975.

12. R. M. Solovay and S. Tennenbaum, Iterated Cohen extensions and Souslin's problem, Ann. of Math. (2) 94 (1971), 201-245.

13. F. D. Tall, On the existence of non-metrizable hereditarily Lindelö spaces with point-countable bases, Duke Math. J. 41 , (1974), 299-304.

14. V. M. Ul'janov, Examples of Lindelö spaces having no compact extensions of countable character, Dokl. Akad. Nauk SSSR 220 (1975), 1282-1285 = Soviet Math. Dokl. 16 (1975), 257-261.

15. L. Vietoris, Bereiche Zweiter Ordnung, Monatsh. Math. 33 (1923), 49-62.

16. S. Willard, General topology, Addison-Wesley, Reading, Mass., 1970.

Department of Mathematics, University of Manitoba, WinNipeg, Mantoba R3T 2N2, CANADA

Current address: Department of Mathematics, University of Toronto, Toronto, Ontario M5S 1A1, Canada 